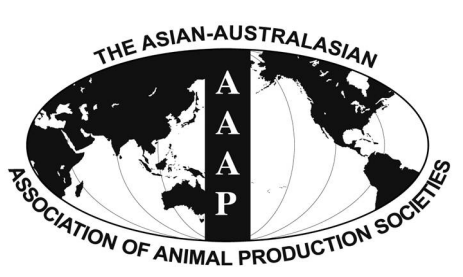

Open Access

Asian Australas. J. Anim. Sci.

Vol. 29, No. 9 : 1239-1246 September 2016

http://dx.doi.org/10.5713/ajas.15.0724

www.ajas.info

pISSN 1011-2367 elSSN 1976-5517

\title{
Comparative Proteomic Analysis of Yak Follicular Fluid during Estrus
}

\author{
Xian Guo*, Jie Pei, Xuezhi Ding, Min Chu, Pengjia Bao, Xiaoyun Wu, Chunnian Liang, and Ping Yan* \\ Key Laboratory of Yak Breeding Engineering of Gansu Province, \\ Lanzhou Institute of Husbandry and Pharmaceutical Sciences, \\ Chinese Academy of Agricultural Sciences, Lanzhou 730050, China
}

\begin{abstract}
The breeding of yaks is highly seasonal, there are many crucial proteins involved in the reproduction control program, especially in follicular development. In order to isolate differential proteins between mature and immature follicular fluid (FF) of yak, the FF from yak follicles with different sizes were sampled respectively, and two-dimensional gel electrophoresis (2-DE) of the proteins was carried out. After silver staining, the Image Master 2D platinum software was used for protein analysis and matrix-assisted laser desorption ionization time of flight mass spectrometry (MALDI-TOF-MS) was performed for differential protein identification. The expression level of transferrin and enolase superfamily member 1 (ENOSF1) was determined by Western blotting for verification analysis. The results showed that 2-DE obtained an electrophoresis map of proteins from mature and immature yak FF with high resolution and repeatability. A comparison of protein profiles identified 12 differently expressed proteins, out of which 10 of them were upregulated while 2 were downregulated. Western blotting showed that the expression of transferrin and ENOSF1 was enhanced with follicular development. Both the obtained protein profiles and the differently expressed proteins identified in this study provided experimental data related to follicular development during yak breeding seasons. This study also laid the foundation for understanding the microenvironment during oocyte development. (Key Words: Yak, Follicular Fluid, Two-dimensional Gel Electrophoresis, Mass Spectrometry, Western Blot)
\end{abstract}

\section{INTRODUCTION}

Yaks (Bos grunniens) are mainly distributed in the Qinghai-Tibetan Plateau and nearby regions; they provide meat, milk, wool and fuel for Tibetan and nomadic pastoralists. These animals are essential for agricultural production in high altitude environment regions. Their natural habitat ranges from between 2,500 to $6,000 \mathrm{~m}$ above sea level. Yaks are seasonally polyestrous, exhibiting cyclic ovarian activity and estrus from July to November (Sarkar and Prakash, 2005; Sarkar et al., 2008). The length of the estrous cycle lasts from 19 to 21 days. However, yaks live in an extremely harsh environment characterized by low temperature, low oxygen content, and high altitude; seasonal nutritional deficiencies seriously affect their

\footnotetext{
* Corresponding Authors: Xian Guo. Tel: +86-931-2115257, Fax: +86-931-2115191, E-mail: guoxian@caas.cn / Ping Yan. Tel: +86931-2115288, Fax: +86-931-2115191, E-mail: yanping@caas.cn Submitted Sept. 1, 2015; Revised Nov. 9, 2015; Accepted Dec. 18, 2015
}

reproductive efficiency (Liu et al., 2012). Unlike bovine females, yak cows rarely express estrus immediately after post-calving during the breeding season. The annual pregnancy rate of most yak breeds is $40 \%$ to $60 \%$, as only a small proportion of the cows return to estrus in the first breeding season after calving; most come into estrus in the second or third years under traditional management systems $(\mathrm{Zi}, 2003)$. These long postpartum anestrous intervals eventually result in poor reproductive efficiency.

Follicular fluid (FF) is a liquid in the ovarian follicle which fills the follicular antrum and surrounds the ovum. FF contains several blood plasma protein components derived from constituents that cross the blood-follicular barrier and several granulosa/theca proteins derived from granulosa and thecal cells (Nandedkar et al., 1992; Fortune, 1994). Thus, FF components may also provide information related to ovarian follicle development. Follicle development is closely associated with yak reproduction and FF provides a very important microenvironment for 
oocyte development. A previous analysis of FF protein composition aimed to identify molecules which may help follicular development and therefore assist in oocyte selection processes (Schweigert et al., 2006). The study of FF protein components have been made by detecting specific proteins and correlating them to estrus and oocyte quality. Recently, more complex molecular techniques have been introduced, allowing for simultaneous study of dozens of proteins and peptides in biological fluid, in a field known as "proteomics" (Revelli et al., 2009).

Protein composition is very complicated and dynamic, and is therefore a major problem in proteomic analysis of complex biological fluids; consequently, sensitive and highresolution protein separation techniques are needed. The most popular FF proteonomic approach is presently based on two-dimensional gel electrophoresis (2-DE) followed by protein digestion and mass spectrometry (Görg et al., 2004). At the same time, Western blotting has been used to detect specific proteins in an extracted sample. Recently, easier techniques have been proposed and applied towards studying FF proteins based on protein prefractionation by isoelectric focusing (IEF) as well as nanoliquid chromatography and mass spectrometry (Hanrieder et al., 2008). In order to improve the efficiency of yak breeding, comparative FF proteomics was applied to analyze different follicular diameters during estrus. This technical scheme involved 2-DE in combination with matrix-assisted laser desorption ionization time of flight mass spectrometry (MALDI-TOF-MS) and Western blotting.

\section{MATERIALS AND METHODS}

\section{Collection of follicular fluid}

Yak ovaries were obtained from a designed abattoir after slaughter in September 2014 in Qinghai Province of China. Sixty ovaries in total were collected from 30 nonpregnant yaks. The yaks were between 6 to 7 years of age, healthy, and free from any anatomical reproductive disorders; they resided 3,200 $\mathrm{m}$ above sea level. The FF was directly aspirated from the ovaries by a sterile syringe and then divided into two groups according to follicular diameter ( 0 to $10 \mathrm{~mm},>10 \mathrm{~mm}$ ), and mixed according to size. The FF was immediately centrifuged at 5,000 $\mathrm{g}$ for 15 min in order to remove blood cells and debris at room temperature. Then, the fluid supernatants were centrifuged a second time at $12,000 \mathrm{~g}$ for $10 \mathrm{~min}$ to remove cells and fractionated at room temperature; the fluid supernatants were directly collected and frozen in liquid nitrogen for further analysis.

\section{Protein extraction and quantitative analysis}

Total FF protein extracts were prepared according to the phenol extraction method described by Zhou et al. (2011) with minor modifications. The most abundant serum proteins were eliminated using the ProteoExtract Albumin/IgG Removal Kit (Calbiochem, San Diego, CA, USA). Total protein concentrations were measured by the Bradford method (Bradford, 1976). Samples were prepared in triplicates and stored at $-80^{\circ} \mathrm{C}$ until use.

\section{Two-dimensional gel electrophoresis}

For 2-DE, $100 \mu \mathrm{g}$ and $400 \mu \mathrm{g}$ of proteins were loaded onto analytical and preparative gels, respectively. The Ettan IPGphor Isoelectric Focusing System (GE Amersham, Amersham, UK) and $\mathrm{pH} 3$ to 10 immobilized $\mathrm{pH}$ gradient (IPG) strips (13 cm, nonlinear; GE Healthcare, Little Chalfont, UK) were used for IEF. The IPG strips were rehydrated for $12 \mathrm{~h}$ in $250 \mu \mathrm{L}$ of rehydration buffer containing the protein samples. The IEF was performed in four steps: $30 \mathrm{~V}$ for $12 \mathrm{~h}, 500 \mathrm{~V}$ for $1 \mathrm{~h}, 1,000 \mathrm{~V}$ for $1 \mathrm{~h}$, and $8,000 \mathrm{~V}$ for $8 \mathrm{~h}$. The gel strips were prepared for $15 \mathrm{~min}$ in equilibration buffer ( $50 \mathrm{mM}$ Tris- $\mathrm{HCl}$ [pH 8.8], $6 \mathrm{M}$ urea, $2 \%$ sodium dodecyl sulfate [SDS], $30 \%$ glycerol, and $1 \%$ DL-dithiothreitol [DTT]). This step was repeated using the same buffer with $4 \%$ iodoacetamide in place of $1 \%$ DTT. The strips were then subjected to the 2-DE after transferring onto $12.5 \%$ SDS-polyacrylamide gels. Electrophoresis was then performed using the Hofer SE 600 system (GE Amersham, UK) at $15 \mathrm{~mA}$ per gel for $30 \mathrm{~min}$, next followed by $30 \mathrm{~mA}$ per gel, until the bromophenol blue reached the end of the gel. Three replicates were performed for each of the samples.

\section{Gel staining and image analysis}

Protein spots in the analytical gels were visualized by silver staining. The preparative gels were stained by a modified silver staining method compatible with subsequent mass spectrometric analysis (Yan et al., 2000). The stained gels were scanned using an UMax Powerlook 2110XL (UMax, Taipei, Taiwan), and the image analysis was accomplished using ImageMaster 2D Platinum (Version 5.0, GE Amersham, UK). Each paired spot was manually verified to ensure a high reproducibility level between the normalized spot gel volumes produced in triplicate data. Two spots were considered as significantly different if $\mathrm{p}<0.05$ and proteins with $\mathrm{a} \geq 1.2 \mathrm{x}$ or greater overlap ratio threshold filtering were considered to be differentially expressed. Spots met the criteria were then selected and subjected to in-gel tryptic digestion. There were $30 \%$ to $35 \%$ higher cutoff points to be chosen for a silver stained gel.

\section{Peptide identification}

All the differentially expressed spots were selected and excised manually from the three preparative gels. Protein spots of interest were cut from the preparative gels, 
destained for $20 \mathrm{~min}$ in $30 \mathrm{mM}$ potassium ferricyanide/100 $\mathrm{mM}$ sodium thiosulfate $(1: 1 \mathrm{v} / \mathrm{v})$ and washed with Milli-Q water until the gels were destained. The spots were incubated in $0.2 \mathrm{M} \mathrm{NH} \mathrm{NHCO}_{3}$ for $20 \mathrm{~min}$ and then lyophilized. Each spot was digested overnight in $12.5 \mathrm{ng} / \mu \mathrm{L}$ trypsin in $25 \mathrm{mM} \mathrm{NH} \mathrm{HCO}_{3}$. The peptides were extracted three times with $60 \%$ acetonitrile $/ 0.1 \%$ trifluoroacetic acid. The extracts were pooled and dried completely by a vacuum centrifuge. The peptides were identified using MASCOT software (Matrix Science, Boston, MA, USA) by searching against the NCBI database (Bos Taurus). If the protein score coverage index (CI) was above $95 \%$ and protein score was above 60 , then it was validated.

\section{MALDI-TOF/TOF MS analysis}

MS (Mass) and MS/MS data used for protein identification were obtained by using a MALDI-TOF-TOF instrument (4800 proteomics analyzer; Applied Biosystems, Waltham, MA, USA). Instrument parameters were set using 4000 Series Explorer software (Applied Biosystems, USA). The MS spectra were recorded in a reflector mode over a mass range from 800 to 4,000 with a focus mass of 2,000 . MS used a CalMix5 standard to calibrate the instrument (ABI 4700 Calibration Mixture). For one main MS spectrum, 25 subspectra (with 125 shots per subspectrum) were accumulated using a random search pattern. For the MS calibration, autolysis peaks of trypsin $([\mathrm{M}+\mathrm{H}]+842.5100$ and 2,211.1046) were used as internal calibrates; up to 10 of the most intense ion signals were selected as MS/MS acquisition precursors, excluding the trypsin autolysis peaks and the matrix ion signals. In MS/MS positive ion mode, one main MS spectrum accumulated 50 subspectra (with 50 shots per subspectrum) using a random search pattern. Collision energy was $2 \mathrm{kV}$, the collision gas was air, and default calibration was set by using the Glu1-Fibrino-peptide B $([\mathrm{M}+\mathrm{H}]+1,570.6696)$ which was spotted onto Cal 7 positions of the MALDI target. Combined peptide mass fingerprinting PMF and MS/MS queries were then performed by using the MASCOT search engine 2.2 (Matrix Science, Ltd., London, UK) embedded into GPS-Explorer Software 3.6 (Applied Biosystems, USA) on the NCBI database (Bos taurus) with the following parameter settings: $100 \mathrm{ppm}$ mass accuracy, trypsin cleavage with one missed cleavage allowed, carbamidomethylation set as fixed modification, methionine oxidation allowed as variable modification, and a MS/MS fragment tolerance set to $0.4 \mathrm{Da}$. A GPS Explorer protein confidence index $\geq 95 \%$ were then used for further manual validation.

\section{Western blot analysis}

Yak FF was homogenized for protein extraction in an ice-cold protein extraction buffer, which was divided into three groups according to follicular diameter ( 0 to $5 \mathrm{~mm}, 5$ to $10 \mathrm{~mm},>10 \mathrm{~mm}$ ). Homogenates were centrifuged for 20 min at $13,000 \mathrm{~g}$ and supernatants were collected. The protein concentrations were determined by the Bradford method. Western blot analysis was performed as described in Moritz et al. (2014) with some modifications. About 300 $\mu \mathrm{g}$ of protein from each sample was denatured, electrophoresed, and transferred onto a polyvinylidene fluoride (PVDF) membrane. The membrane was blocked and blots were intubated in a specific antibody against enolase superfamily member 1 (ENOSF1) and transferrin, followed by secondary antibodies according to manufacturer. The NBT/BCIP system was used to evaluate the protein signal.

\section{Immunoblotting}

The proteins were separated by sodium dodecyl sulfate polyacrylamide gel electrophoresis (SDS-PAGE) on 10\% polyacrylamide gels. Polypeptides were then transferred to PVDF membranes $(0.22 \mu \mathrm{m}$, Amersham Life Science, Little Chalfon, UK) in a medium which consisted of $25 \mathrm{mM}$ Tris$\mathrm{HCl}(\mathrm{pH} \mathrm{8.3)}, 192 \mathrm{mM}$ glycine, and 20\% (v/v) methanol. After rinsing in the tris-buffered saline (TBS) which contained $10 \mathrm{mM}$ Tris- $\mathrm{HCl}(\mathrm{pH} 7.5)$ and $150 \mathrm{mM} \mathrm{NaCl}$, the blotted membranes were pre-incubated for $2 \mathrm{~h}$ in a blocking buffer containing $5 \%(\mathrm{w} / \mathrm{v})$ non-fat milk dissolved in TBS supplemented by $0.05 \%$ (v/v) Tween-20 (TBST1). The components were then incubated by gentle shaking for $2 \mathrm{~h}$ at room temperature in the appropriate antibodies (antiENOSF1, rabbit polyclonal antibodies to ENOSF1 [ab182354; Abcam, Cambridge, MA, USA]) diluted 1:2000 in the blocking buffer; anti-transferrin (sheep polyclonal antibodies to transferrin [ab112892; Abcam, USA] diluted $1: 1,000$ in the blocking buffer; anti-actin diluted $1: 1,000$ in the blocking buffer). Following extensive washes by TBST1, the membranes were then incubated with Goatanti-Rabbit $\mathrm{IgG}$ conjugated with either horseradish peroxidase (1:5,000 diluted in TBST1) alone or Rabbit-antiMouse IgG conjugated with horseradish peroxidase (1:5,000 diluted in TBST1) at room temperature for $2 \mathrm{~h}$. Afterwards, the substance was washed with TBST2 (50 mM Tris-HCl, pH 7.5, $150 \mathrm{mM} \mathrm{NaCl}, 0.1 \%$ [v/v] Tween-20) and TBS. Locations of antigenic proteins were visualized by scanning the membranes with the ImageQuant LAS 500 machine (GE Healthcare, UK).

\section{Statistical analysis}

The homogeneity of variance was checked by using Levene's test. If the data failed to pass the test, then a logarithmic transformation was used. The differences were evaluated by a one-way analysis of variance followed by a 

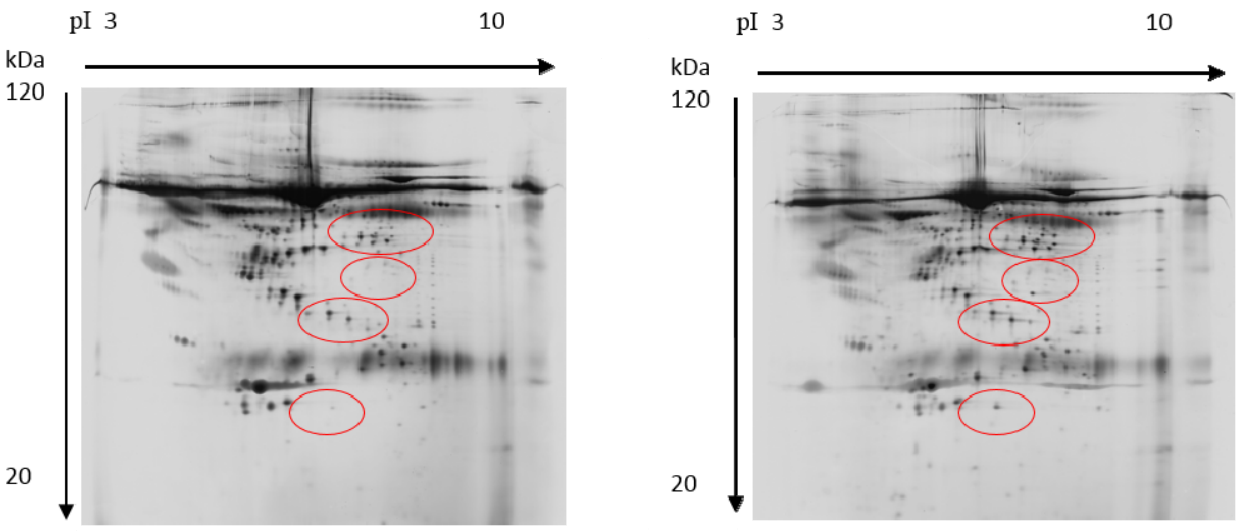

Figure 1. Two-dimensional gel electrophoresis (2-DE) of mature (Left) and immature (Right) follicular fluid. Selected protein spots were numbered and collected for identification by matrix-assisted laser desorption ionization time of flight mass spectrometry (MALDI-TOFMS).

Tukey's test using SPSS 13.0 (SPSS, Chicago, IL, USA). bioinformatic analysis, up to 12 differentially expressed Significant differences between mature and immature FF were identified by a p-value of 0.05 .

\section{RESULTS}

Comparative proteome analysis by two-dimensional gel electrophoresis

Global protein components of yak FF were separated with high sensitivity and resolution by 2-DE; more than 100 spots were detected for each gel. Spots with a significant increase or decrease in relative abundance were considered to represent differently expressed proteins. After proteins were obtained; 10 expressed proteins were found more abundantly in the mature FF, while 2 expressed proteins were upregulated in the immature FF (Figure 1). Some of them were found to have the same isoelectric points (pI) and molecular weights (MW) as predicted in the database.

\section{Identification of differently expressed proteins}

Differently expressed proteins were picked from gels and identified using MS/MS. Figure 2 shows typical examples of some differently expressed proteins identified by 2-DE. All data from the conventional gel-to-gel

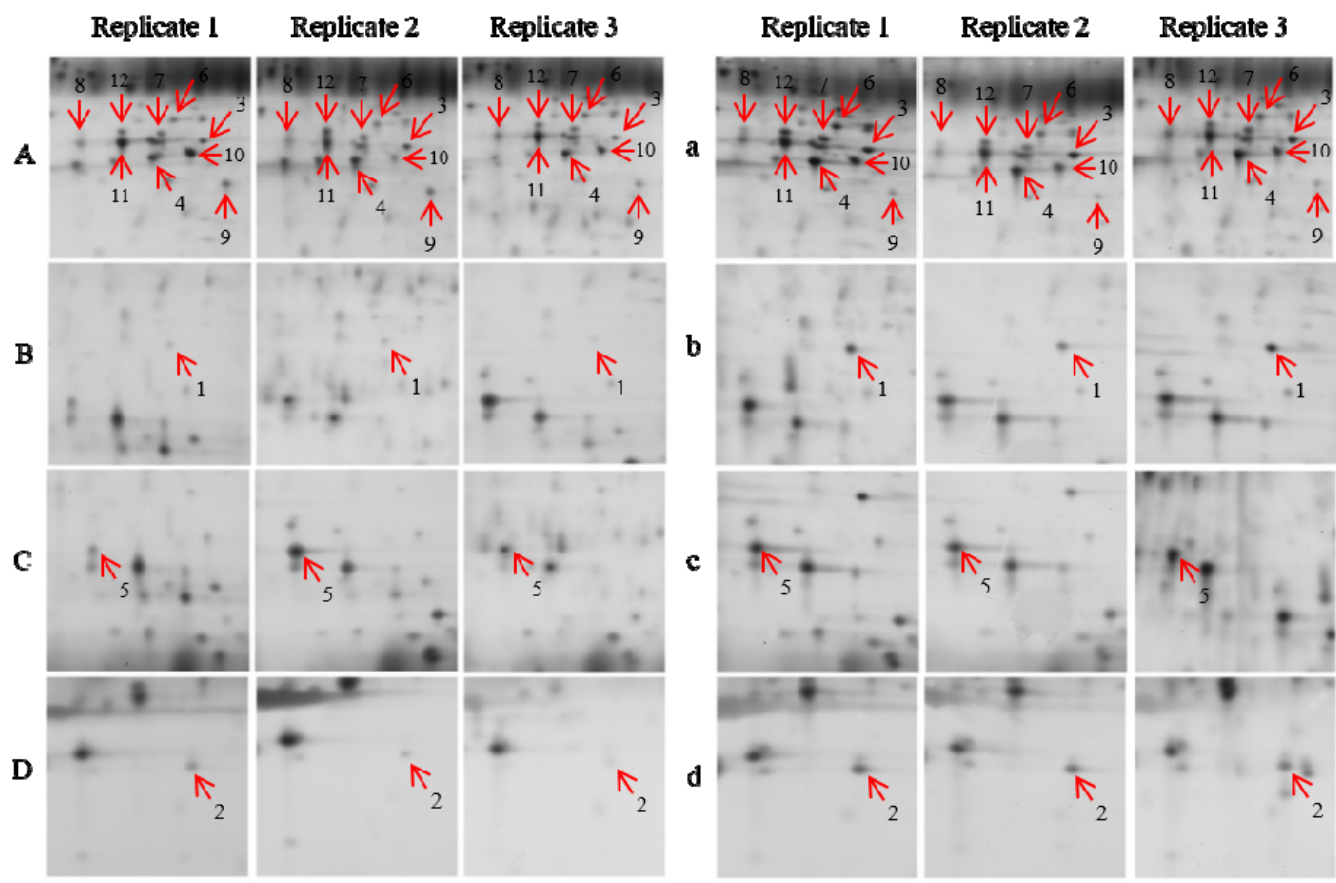

Figure 2. Typical examples of some differently expressed protein spots with at least two-fold changes in mature (A, B, C, D) and immature $(\mathrm{a}, \mathrm{b}, \mathrm{c}, \mathrm{d})$ yak follicular fluid by two-dimensional electrophoresis. Replicate 1, 2, and 3 images in the figure were triplicate gels. 
Table 1. Matrix-assisted laser desorption/ionization time of flight mass spectrometry (MALDI-TOF-MS/MS) identification of differently expressed proteins in the yak follicular fluid

\begin{tabular}{|c|c|c|c|c|c|c|c|}
\hline NO. & Protein name & Accession No. & $\begin{array}{l}\text { Protein } \\
\text { MW }\end{array}$ & $\begin{array}{l}\text { Protein } \\
\text { PI }\end{array}$ & $\begin{array}{l}\text { Pep. } \\
\text { count }\end{array}$ & $\begin{array}{l}\text { Protein } \\
\text { score }\end{array}$ & $\begin{array}{l}\text { Protein } \\
\text { score } \mathrm{CI} \%\end{array}$ \\
\hline 1 & Serotransferrin precursor [Bos taurus] & gi|114326282 & $79,855.5$ & 6.75 & 26 & 386 & 100 \\
\hline 2 & Unnamed protein product [Bos taurus] & gi|110292444 & $71,264.2$ & 5.82 & 30 & 600 & 100 \\
\hline 3 & Serum albumin precursor $[$ Bos taurus $]$ & gi|30794280 & $71,274.2$ & 5.82 & 28 & 220 & 100 \\
\hline 4 & $\begin{array}{l}\text { Chain A, crystal structure of bovine serum albumin in } \\
\text { complex with 3,5- diiodosalicylic acid [Bos taurus] }\end{array}$ & gi|529482051 & $68,415.6$ & 5.6 & 16 & 692 & 100 \\
\hline 5 & Mitochondrial enolase superfamily member 1 [Bos taurus] & gi|114052721 & $50,216.4$ & 5.86 & 13 & 89 & 100 \\
\hline 6 & TPA: alpha-enolase [Bos taurus] & gi|296479148 & $47,596.5$ & 6.37 & 15 & 111 & 100 \\
\hline 7 & TPA: serum albumin precursor $[$ Bos taurus $]$ & gi|296486410 & $71,274.2$ & 5.82 & 18 & 101 & 100 \\
\hline 8 & transferrin $[$ Bos taurus $]$ & gi|602117 & $79,869.5$ & 6.75 & 16 & 79 & 99.852 \\
\hline 9 & PREDICTED: fibrinogen beta chain isoform X1 [Bos taurus] & gi|528981280 & $56,349.6$ & 8.5 & 13 & 107 & 100 \\
\hline 10 & Deoxyribonuclease II beta [Bos taurus] & gi|109939926 & $38,864.2$ & 9.06 & 8 & 69 & 98.594 \\
\hline 11 & $\begin{array}{l}\text { PREDICTED: C-type lectin domain family } 9 \text { member A-like } \\
\text { isoform X1 [Bos taurus] }\end{array}$ & gi|528950700 & $30,415.5$ & 9.23 & 12 & 60 & 100 \\
\hline 12 & Tetranectin precursor $[$ Bos taurus $]$ & gi|114051137 & $22,586.1$ & 5.47 & 4 & 93 & 99.994 \\
\hline
\end{tabular}

MW, molecular weights; PI, isoelectric point; CI, coverage index.

experiments are summarized in Table 1. Proteins with MW ranging from 20 to $120 \mathrm{kDa}$ and $\mathrm{pI}$ between 3 and 10 were well-separated. Twelve significantly and consistently up- or downregulated proteins spots with a twofold change in volume intensity in the triplicate gels were trypsin-digested and analyzed by MALDI-TOF-MS/MS.

The following three criteria were used for the identification of specific proteins: i) Several peptides were found to be specific for a given protein; ii) At least $12 \%$ of the amino acid sequence of protein was covered; iii) Observed MW and pI determined for a protein by 2-DE gel electrophoresis agreed with calculated values. Based on these criteria, 34 protein spots were successfully identified that corresponded to 14 proteins; all of these 12 proteins matched well with bovine proteins. Among these 12 proteins, 10 of them were upregulated in mature yak FF, including transferrin and ENOSF1. In comparison, the other 2 proteins were downregulated in immature yak FF.

\section{Analysis of differently expressed proteins}

The function and MW of 12 Bos taurus proteins were analyzed according to the NCBI database. From the

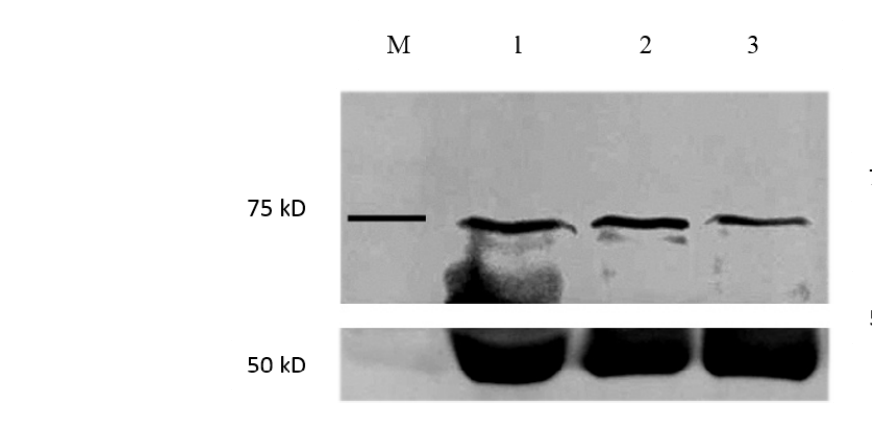

candidates, ENOSF1 and transferrin were selected for Western blot analyses due to their correlation with animal reproduction. As shown in Figure 3, the changes in expression of both selected proteins was consistent with the 2-DE and silver-staining results. These results demonstrated that the proteomic analysis of FF was a convincing assessment of reproduction response.

\section{DISCUSSION}

Yaks have seasonal polyestrous cycles with breeding that occurs from June to November; the largest proportion of female yaks come into estrus in the middle of this period (July to September) in most yak production areas ( $\mathrm{Zi}, 2003$; Sarkar and Prakash, 2005). Reproduction in yaks is low as a result of seasonal breeding, delayed puberty, and lower estrus frequency. Therefore, numerous recent studies have focused on reproduction and physiology in yaks, as well as assisted reproductive techniques (Sarkar et al., 2008; Guo et al., 2012). Follicular development is a very complicated process; it plays a role in oocyte maturation and the ovulation mechanism during yak breeding season.

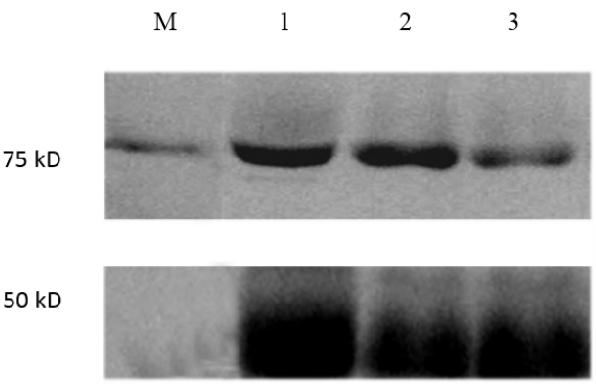

Figure 3. Antibody signals for transferrin and ENOSF 1 obtained from there different follicular diameters ( 0 to $5 \mathrm{~mm}, 5$ to $10 \mathrm{~mm},>10$ $\mathrm{mm})$. M: Marker, 1: Follicular diameter $>10 \mathrm{~mm}, 2$ : Follicular diameter between $5 \mathrm{~mm}$ and $10 \mathrm{~mm}, 3$ : Follicular diameter $<5 \mathrm{~mm}$. 
Proteomics is a significant focus of life science studies in the post-genomic era. Proteomics-based studies examine the overall protein expression in tissues or cells, increasing the possibility of answering more complex biological questions. In addition, mass spectrometry techniques have enabled scientists to carry out molecular analysis of peptides and proteins in a sensitive, rapid, and specific manner; they have fundamentally transformed the biological and biochemical study of proteomics (Han et al., 2008). Therefore, an overall analysis of proteomics combined with spectrometry can alleviate challenges in assessing the complex FF composition (Upadhyay et al., 2013). Proteomic-based analysis of FF protein changes during yak follicular development helps to identify proteins that either possibly participate or are even key to follicular development and oocyte maturation; it also helps to determine potential regulatory mechanisms and yak seasonal breeding at a proteome level. Thus, proteomicbased analysis provides new insights for improving yak ovarian reserve capacity and reproductive performance.

Proteins associated with follicle and oocyte maturation and development can be identified by using the proteomic approaches comparing protein profiles in different developmental stages of FF. Spitzer et al. (1996) carried out 2-DE to compare protein profiles between mature and immature human follicles; their results indicated different FF protein contents in different developmental stages could be used as follicular maturation biomarkers. Liu et al. (2007) performed MALDI-TOF-MS to analyze specific FF peptides of different developmental stages by hydrolyzing FF proteins and analyzing final products using MALDIFOL-MS. A comparison of peaks obtained from samples at different development stages identified 5 specific peaks with a $100 \%$ occurrence in mature oocyte $\mathrm{FF}$ and 25 specific peaks with a $100 \%$ occurrence in immature oocyte FF. By searching the protein database, numerous proteins associated with oocyte maturation were identified. In this study, in order to investigate proteomic changes occurring in FF between mature and immature yak oocyte follicles, FF proteomes were analyzed using the 2-DE and MALDITOF-MS approaches. In total, 34 different expressed protein spots were obtained, 12 of which were successfully identified to correspond with bovine proteins. The 12 proteins selected spots were identified as unique proteins, 9 of which were validated (protein score $>100$ ). A comparison of protein profiles identified 12 differently expressed proteins, out of which 10 were upregulated while 2 were downregulated. Functional analyses revealed that most of up- or downregulated proteins were both involved in metabolism, binding, signal transduction, cell developmental pathways and cell structure. The study provides a solid basis for futher research into the process that regulates follicular development and oocyte maturation in yak during estrus.

Western blotting is also called immunoblotting, a type of immunochemical technique used to detect a protein immobilized on a matrix. This study used Western blotting to detect transferrin and ENOSF1 expression. Our results suggested transferrin and ENOSF1 expression were enhanced with follicular development ( 0 to $5 \mathrm{~mm}, 5$ to 10 $\mathrm{mm},>10 \mathrm{~mm}$ ).

\section{Eenolase superfamily member 1}

The enolase superfamily (ENOSF), named after the enolase enzyme of glycolysis, is used as a model of protein superfamily evolution (Gerlt et al., 2005). The enolase superfamily members share a common ENOSF fold and catalyze a common half reaction: they all abstract protons that are adjacent to carboxyl groups from numerous substrates. The $E N O S F 1$ gene was originally identified as a naturally occurring human thymidylate synthase (TYMS) gene antisense transcript (Dolnick, 1993) and codes for two proteins (rTS $\alpha$ and rTS $\beta$ ) through alternative RNA splicing (Dolnick, 1993). The ENOSF1 gene function appears primarily to regulate TYMS locus expression both via the antisense transcript and through encoded proteins (Dolnick et al., 2003). Evolutionarily conserved acidic residues, in loops located at the end of two $\beta$-sheets lining the ENOSF fold C-terminal barrel, coordinate with an essential magnesium; they are shared by all ENOSFs. Within the superfamily, different ENOSF families are distinguished by the identity of the third magnesium ligand and the different general acid/base catalytic residue combinations at the ends of the remaining $\beta$ sheets in the C-terminal barrel (Gerlt et al., 2005). In addition, Finckbeiner (2011) reported ENOSF1 function in vertebrates, showing that ENOSF1 is required for embryonic development. The increased apoptosis following ENOSF1b knockdown suggests a potential survival advantage for increased ENOSF1 $\beta$ expression in human cancers. Our study showed that the ENOSF1 expression level in mature yak FF was higher than in immature yak FF. The protein level increased along with follicle development, indicating ENOSF1 may play a regulatory role and/or can be considered a biomarker for yak follicular development. However, its possible role in a regulating mechanism requires further investigation.

\section{Transferrin}

Transferrin is the main iron transport protein found in the circulation. The transferrin saturation level in the blood is an important indicator of iron status (Kovac et al., 2009). Transferrin is can bind two $\mathrm{Fe}^{3+}$ ions with very high affinity and donate iron to cells throughout the body via transferrin receptor 1 (TfR1) (Kovac et al., 2009). The crystal structure of the single transferrin polypeptide chain (which is comprised of 680 to 690 amino acid residues) has been 
determined in both the diferric (Bailey et al., 1988) and iron-free (apo-transferrin) forms (Wally et al., 2006). The chain is folded into two lobes (the N-lobe and C-lobe) derived from the $\mathrm{N}$-terminal and $\mathrm{C}$-terminal halves of the protein, respectively. The two lobes share $60 \%$ homology and are presumed to arise from gene duplication and fusion (Park et al., 1985). Each lobe is folded into two subdomains which come together to form a cleft, providing a binding site for one ferric ion (Baker et al., 2003). In the serum-free culturing of follicular granulosa cells, transferrin is often added as a growth factor into the medium to stimulate the cell growth and development (Orly et al., 1980). In this study, MALDI-TOF-MS-based isolation and identification of immature and mature yak FF found that transferrin was significantly expressed in mature FF; the protein level increased along with yak follicle development. This result indicated transferrin may significantly stimulate follicular maturation and therefore be a potential biomarker for yak follicular maturation.

\section{CONCLUSION}

In the breeding season, expression of different proteins in yak FF may vary over different developmental stages. This study identified 12 differently expressed proteins, of which 10 exhibited upregulated expression and 2 had downregulated expression during follicular maturation. Western blotting showed that transferrin and ENOSF1 expression were enhanced with follicular development. These differently expressed proteins may regulate and influence follicular maturation according to their level of function.

\section{CONFLICT OF INTEREST}

We certify that there is no conflict of interest with any financial organization regarding the material discussed in the manuscript.

\section{ACKNOWLEDGMENTS}

The work was supported or partly supported by grants from National Natural Science Foundation of China (31301976), Science and Technology Support Projects in Gansu Province (1504NKCA052), China Agriculture Research System (CARS-38), National Science and Technology Support Project (2013BAD16B09), and the Innovation Project of Chinese Academy of Agricultural Sciences (CAAS-ASTIP-2014-LIHPS-01).

\section{REFERENCES}

Bailey, S., R. W. Evans, R. C. Garratt, B. Gorinsky, S. Hasnain, C.
Horsburgh, H. Jhoti, P. F. Lindley, A. Mydin, and R. Sarra et al. 1988. Molecular structure of serum transferrin at 3.3-A resolution. Biochemistry 27:5804-5812.

Baker, H. M., Q. Y. He, S. K. Briggs, A. B. Mason, and E. N. Baker. 2003. Structural and functional consequences of binding site mutations in transferrin: Crystal structures of the Asp63Glu and Arg124Ala mutants of the N-lobe of human transferrin. Biochemistry 42:7084-7089.

Bradford, M. M. 1976. A rapid and sensitive method for the quantitation microgram quantities of protein utilizing the principle of protein-dye binding. Anal. Biochem. 72:248-254.

Dolnick, B. J., N. J. Angelino, R. Dolnick, and J. R. Sufrin. 2003. A novel function for the $r T S$ gene. Cancer Biol. Ther. 2:364369.

Dolnick, B. J. 1993. Cloning and characterization of a naturally occurring antisense RNA to human thymidylate synthase mRNA. Nucleic Acids Res. 21:1747-1752.

Finckbeiner, S., P. J. Ko, B. Carrington, R. Sood, K. Gross, B. Dolnick, J. Sufrin, and P. Liu. 2011. Transient knockdown and overexpression reveal a developmental role for the zebrafish enosflb gene. Cell Biosci. 1:32.

Fortune, J. E. 1994. Ovarian follicular growth and development in mammals. Biol. Reprod. 50:225-232.

Gerlt, J. A., P. C. Babbitt, and I. Rayment. 2005. Divergent evolution in the enolase superfamily: the interplay of mechanism and specificity. Arch. Biochem. Biophys. 433:5970 .

Guo, X., X. Z. Ding, J. Pei, P. J. Bao, C. N. Liang, M. Chu, and P. Yan. 2012. Efficiency of in vitro embryo production of yak (Bos grunniens) cultured in different maturation and culture conditions. J. Appl. Anim. Res. 40:323-329.

Görg, A., W. Weiss, and M. J. Dunn. 2004. Current twodimensional electrophoresis technology for proteomics. Proteomics 4:3665-3685.

Han, X., A. Aslanian, and J. R. Yates. III. 2008. Mass spectrometry for proteomics. Curr. Opin. Chem. Biol. 12:483-490.

Hanrieder, J., A. Nyakas, T. Naessén, and J. Bergquist. 2008. Proteomic analysis of human follicular fluid using an alternative bottom-up approach. J. Proteome Res. 7:443-449.

Kovac, S., A. Ferrand, J. P. Esteve, A. B. Mason, and G. S. Baldwin. 2009. Definition of the residues required for the interaction between glycine-extended gastrin and transferrin in vitro. FEBS J. 276:4866-4874.

Liu, A. X., Y. M. Zhu, Q. Luo, Y. T. Wu, H. J. Gao, X. M. Zhu, C. M. Xu, and H. F. Huang. 2007. Specific peptide patterns of follicular fluids at different growth stages analyzed by matrixassisted laser desorption/ionization time-of-flight mass spectrometry. Biochim. Biophys. Acta 1770:29-38.

Liu, B., H. Zhang, M. Hao, and S. Yu. 2012. Establishment and characterization of two fetal fibroblast cell lines from the yak. In Vitro Cell. Dev. Biol. Anim. 48:619-624.

Moritz, C. P., S. X. Marz, R. Reiss, T. Schulenborg, and E. Friauf. 2014. Epicocconone staining: A powerful loading control for Western blots. Proteomics 14:162-168.

Nandedkar, T. D., J. K. Shahid, R. Mehta, S. B. Moodbidri, U. C. Hegde, and I. N. Hinduja. 1992. Localization and detection of ovarian follicular fluid protein in follicles of human ovaries. Indian J. Exp. Biol. 30:271-275. 
Orly, J., G. Sato, and G. F. Erickson. 1980. Serum suppresses the expression of hormonally induced function in cultured granulosa cells. Cell 20:817-827.

Park, I., E. Schaeffer, A. Sidoli, F. E. Baralle, G. N. Cohen, and M. M. Zakin. 1985. Organization of the human transferrin gene: Direct evidence that it originated by gene duplication. Proc. Natl. Acad. Sci. USA. 82:3149-3153.

Revelli, A., L. D. Piane, S. Casano, E. Molinari, M. Massobrio, and P. Rinaudo. 2009. Follicular fluid content and oocyte quality: from single biochemical markers to metabolomics. Reprod. Biol. Endocrinol. 7:40.

Sarkar, M. and B. S. Prakash. 2005. Synchronization of ovulation in yaks (Poephagus grunniens L.) using PGF2 $\alpha$ and GnRH. Theriogenology 63:2494-2503.

Sarkar, M., D. H. Sengupta, B. D. Bora, J. Rajkhoa, S. Bora, S. Bandopadhaya, M. Ghosh, F. A. Ahmed, P. Saikia, K. Mohan, and B. S. Prakash. 2008. Efficacy of heatsynch protocol for induction of estrus, synchronization of ovulation and timed artificial insemination in yaks (Poephagus grunnies L.). Anim. Reprod. Sci. 104:299-305.

Schweigert, F. J., B. Gericke, W. Wolfram, U. Kaisers, and J. W. Dudenhausen. 2006. Peptide and protein profiles in serum and follicular fluid of women undergoing IVF. Hum. Reprod. 21:2960-2968.
Spitzer, D., K. F. Murach, F. Lottspeich, A. Staudach, and K. Illmensee. 1996. Different protein patterns derived from follicular fluid of mature and immature human follicles. Hum. Reprod. 11:798-807.

Upadhyay, R. D., N. H. Balasinor, A. V. Kumar, G. Sachdeva, P. Parte, and K. Dumasia. 2013. Proteomics in reproductive biology: Beacon for unraveling the molecular complexities. Biochim. Biophys. Acta 1834:8-15.

Wally, J., P. J. Halbrooks, C. Vonrhein, M. A. Rould, S. J. Everse, A. B. Mason, and S. K. Buchanan. 2006. The crystal structure of iron-free human serum transferrin provides insight into inter-lobe communication and receptor binding. J. Biol. Chem. 281:24934-24944.

Yan, J. X., R. Wait, T. Berkelman, R. A. Harry, J. A. Westbrook, C. H. Wheeler, and M. J. Dunn. 2000. A modified silver staining protocol for visualization of proteins compatible with matrixassisted laser desorption/ionization and electrospray ionization-mass spectrometry. Electrophoresis 21:3666-3672.

Zhou, D. H., Z. G. Yuan, F. R. Zhao, H. L. Li, Y. Zhou, R. Q. Lin, F. C. Zou, H. Q. Song, M. J. Xu, and X. Q. Zhu. 2011. Modulation of mouse macraophage proteome induced by Toxoplasma gondii tachyzoites in vivo. Parasitol. Res. 109:1637-1646.

Zi, X. D. 2003. Reproduction in female yaks (Bos grunniens) and opportunities for improvement. Theriogenology 59:1303-1312. 